\title{
Die Arbeitslosigkeitsfalle vor und nach der Hartz-IV-Reform
}

\author{
Sonja Fehr \\ Georg Vobruba
}

\begin{abstract}
Ziel der Hartz-Reformen war es, am Übergang zwischen dem System sozialer Sicherung und dem Arbeitsmarkt Grundsätzliches zu ändern: Durch forcierte Aktivierung der Transferbezieher sollten die Integration erwerbsfähiger Grundsicherungsbezieher in den Arbeitsmarkt beschleunigt und staatliche Sozialtransferausgaben begrenzt werden. Die Reformen rekurrierten auf das Armutsfallentheorem der Wirtschaftswissenschaften: Arbeitslose lassen sich durch Lohnersatzleistungen dazu verleiten, keine Suchanstrengungen auf dem Arbeitsmarkt anzustellen und verbleiben im Sozialtransferbezug. Wir fassen dies als eine empirisch prüfbare Hypothese auf und fragen nach der Dauer individueller Arbeitslosigkeit vor und nach der Hartz-IV-Reform. ${ }^{1}$
\end{abstract}

\section{Einleitung}

Die öffentlichen Debatten über die Folgen der Hartz-IV-Reform konzentrieren sich auf zwei Fragen. Zum einen werden die Hartz-Reformen unter Gesichtspunkten von Gerechtigkeit diskutiert. Zum anderen wird danach gefragt, ob die Reformen problemangemessen zugeschnitten sind und zur Lösung von Problemen tatsächlich beigetragen haben. Dabei werden in der öffentlichen Diskussion Gerechtigkeitskosten der Hartz-IV-Reform in der Regel mit ihren Effizienzgewinnen gerechtfertigt. Mit der Frage der Gerechtigkeit der Hartz-IV-Reform befassen wir uns hier nicht. Es geht uns auch nicht um eine möglichst umfassende Evaluierung der Reform (vgl. dazu Koch et al. 2009). Wir konzentrieren uns vielmehr auf eine einzige, aber entscheidende Frage: Ist es mit der Hartz-IV-Reform gelungen, die allgemein als Problem gesehene Arbeitslosigkeitsfalle zwischen Arbeitsmarkt und dem System sozialer Sicherung zu beseitigen und ausreichende Arbeitsanreize für die Bezieher von Lohnersatzleistungen sicherzustellen? Um diese Fragen zu diskutieren, gehen wir in den folgenden Schritten vor:

Zunächst werden wir kurz zeigen, dass es für die Hartz-IV-Reform politisch zentral war, das Anreizproblem für arbeitslose Bezieher von Sozialtransfers in den Griff zu bekommen. Um beurteilen zu können, ob die Reform diesbezüglich erfolgreich war, müssen wir zwei Fragen klären. Zuerst untersuchen wir, ob das Problem einer Arbeitslosigkeitsfalle vor der Einführung von Hartz IV überhaupt bestand. Dazu betrachten wir die Verweildauern erwerbsfähiger Sozial- und Arbeitslosenhilfebezieher (vor Hartz IV) in Arbeitslosigkeit. Dann fragen wir, wie sich die Verweildauern seit Hartz IV im Bezug von Arbeitslosengeld II entwickelt haben. Da wir uns auf arbeitslose Sozialtransferbezieher konzentrieren, übernehmen wir den in der ökonomischen Theorie und insbesondere im englischen Sprachgebrauch üblichen Begriff der „Arbeitslosigkeitsfalle“ (unemployment trap) (vgl. Gebauer/ Vobruba 2003; Gebauer 2007, S. 75ff.).

Mit diesem vergleichenden Ansatz lässt sich einiges erschließen: Zum einen kann man den Erfolg von Hartz IV nur anhand eines Vorher-Nachher-Vergleichs beurteilen. Wie war die Problemlage vor der Reform? Wie hat Hartz IV auf die Übergänge aus dem Grundsicherungsbezug in den Arbeitsmarkt gewirkt und wie haben sich die Übergangschancen durch die Reform verändert? Und zum anderen kann man erst vor diesem Hintergrund sinnvoll diskutieren, ob die Hartz-IV-Reform ihre sozialen Kosten einer Zunahme von sozialer Ungleichheit und Armut, höherer Beschäftigungsunsicherheit sowie Armut trotz Arbeit (working poor) wert war (vgl. Knuth 2006; Buntenbach 2009). Dies ist hier freilich nicht mehr unser Thema.

\section{2 \\ Untersuchungsgegenstand}

Schon in der Agenda 2010, dem programmatischen Politikentwurf, der den Hartz-Reformen vorausging, wurde die Beseitigung von sozialpolitisch bewirkten Beschäftigungshindernissen als zwingende Notwendigkeit bezeichnet: „Die Reformen auf dem Arbeitsmarkt helfen, dass Arbeitsuchende wieder schneller Arbeit finden. Nach dem Prinzip Fördern und Fordern müssen Langzeitarbeitslose jede zumutbare Arbeit annehmen" (Bundesregierung 2004, S. 9). Es ging explizit darum, mehr „Anreiz für die Aufnahme von Arbeit" $\mathrm{zu}$ schaffen und zu verhindern, dass

1 Wir danken den anonymen Gutachtern für ihre Hinweise und Kommentare.

Sonja Fehr, Soziologin, ist wissenschaftliche Mitarbeiterin im Forschungsbereich Erwerbslosigkeit und Teilhabe am Institut für Arbeitsmarkt- und Berufsforschung (IAB) in Nürnberg. Arbeitsschwerpunkte: Arbeitsmarkt-, Jugend- und Bildungssoziologie. e-mail: sonja.fehr@iab.de Georg Vobruba, Prof. Dr., lehrt Sozialpolitik am Institut für Soziologie der Universität Leipzig. Arbeitsschwerpunkte: Soziologie der sozialen Sicherheit, Europasoziologie, soziologische Gesellschaftstheorie. e-mail:vobruba@uni-leipzig.de 
Leute, „die dem Arbeitsmarkt womöglich gar nicht zur Verfügung stehen, Arbeitslosenhilfe beziehen“" (Bundesregierung 2003, S. 5). Dies ist die dominierende Problemsicht der Politik (vgl. auch BMWA 2005) und entspricht den Auffassungen des ökonomischen Mainstream. Die fachökonomische Klage, dass das System sozialer Sicherung durch seine Lohnersatzleistungen für arbeitsfähige Personen Beschäftigung behindere, da es den Arbeitsanreiz reduziere (vgl. Sinn et al. 2002; Schäfer 2003), hat eine lange Tradition. „Nicht weil rationalisiert wird, sondern weil die Arbeitslosen der Notwendigkeit, sich nach neuer Arbeit umzusehen, enthoben werden, gibt es Arbeitslosigkeit als Dauererscheinung". So brandmarkte Ludwig von Mises (1931, S. 23) auf dem Höhepunkt der Weltwirtschaftskrise in den Jahren nach 1929 die Arbeitslosenversicherung als eine Ursache der Massenarbeitslosigkeit. Gegen die anreizstörenden Wirkungen von sozialpolitischen Lohnersatzleistungen wurde das Lohnabstandsgebot formuliert, Arbeitslosigkeit wurde und wird als Ergebnis des Verstoßes gegen dieses Gebot angesehen. „Negative Anreizwirkungen sind vor allem dort zu erwarten, wo zwischen Sozialhilfe und den bei regulärer Tätigkeit zu erzielenden Einkommen kein hinreichender Abstand besteht" (SVR 1998, S. 235). Im gleichen Sinn und unter ausdrücklichem anreiztheoretischem Bezug formuliert die Lehrbuchökonomie: „Da die Leistungen aus der Arbeitslosenversicherung aufhören, sobald der Arbeitslose wieder beschäftigt ist, besteht bisweilen eine geringe Neigung zu nachdrücklicher Suche und die Möglichkeit, unattraktive Stellenangebote auszuschlagen“ (Mankiw 1999, S. 635).

Der infrage stehende Zusammenhang von sozialpolitischen Lohnersatzleistungen, Arbeitsanreizen, Arbeitsbereitschaft und Partizipation am Arbeitsmarkt wird in der internationalen Diskussion unter den Stichworten „Armutsfalle“, „Arbeitslosigkeitsfalle“, „poverty trap“ oder „unemployment trap" besprochen. Arbeitslose Arbeitskräfte im unteren Einkommensbereich - so die These-ließen sich durch vergleichsweise hohe Lohnersatzleistungen dazu verleiten, keine ernsthaften Suchanstrengungen auf dem Arbeitsmarkt anzustellen und freiwillig im Sozialtransferbezug zu verbleiben. Daraus resultierten zwei Effekte: Zum einen reduziere sich mit zunehmender Dauer ihrer Arbeitslosigkeit ihre Chance auf Wiederbeschäftigung immer mehr. Zum anderen bedeute für Arbeitgeber sozialpolitisch induzierte freiwillige Arbeitslosigkeit eine Verknappung des Angebots in diesem Einkommensbereich. Darauf reagierten sie mit Rationalisierungsinvestitionen und reduzierter Nachfrage nach Arbeitskräften. Beide Effekte hätten zur Folge, dass sich die Wiederbeschäftigungschancen von Arbeitskräften im unteren Einkommensbereich verschlechterten und dass sich so anfangs freiwillige Arbeitslosigkeit als unfreiwillige Arbeitslosigkeit verfestige: Die Arbeitslosen gerieten durch ihr eigenes, durch sozialpolitische Fehlanreize hervorgerufenes Verhalten in eine Falle. Dieses Theorem hat politische Folgen.

Die Konzipierung dieses Zusammenhangs als „Falle“ erschließt die Legitimation für weitreichende Interventionsmöglichkeiten. Möglich wird eine Politikattitüde der stellvertretenden Interessenwahrnehmung: Politik gegen die unmittelbaren Interessen der Betroffenen im Namen ihres längerfristigen wohlverstandenen Interesses wird legitim (vgl. Gebauer et al. 2003, S. 13).

Sozialwissenschaftlich bemerkenswert und politisch folgenreich ist der Umstand, dass in der Ökonomie, der ohne Zweifel ein Quasi-Interpretationsmonopol der Arbeitsmarktrealität zukommt, dieser Zusammenhang bisweilen nicht als empirisch überprüfbare Vermutung, sondern als Tatsache formuliert wird (vgl. dazu Vobruba 2000, S. 85ff.). Diese Gewissheit wurde - jedenfalls im Zuge der HartzReformen - als Problemdiagnose in die Politik übernommen.

\section{Forschungsstand}

Die dynamische Armutsforschung setzt dem Armutsfallentheorem empirisch entgegen, dass Armut und Bezug von Sozialleistungen oft eine vorübergehende Episode im Leben von Menschen sind. Bane/Elwood (1986) konnten bereits in den 1980 er Jahren zeigen, dass es eine hohe Fluktuation unter den Beziehern wohlfahrtsstaatlicher Leistungen gibt. Ihre Erkenntnisse wurden von der deutschen Armutsforschung (vgl. Buhr 1995; Leisering/ Leibfried 1999) und von internationalen Studien bestätigt (vgl. Heikkikilä/Keskitalo 2001; Gustafson et al. 2002; Saraceno 2002; Lightman et al. 2010).
Die Zahl an Untersuchungen, welche die These einer Arbeitslosigkeitsfalle im Sinne einer Hypothese auffassen und anhand tatsächlichen Verhaltens empirisch überprüfen, ist indes überschaubar (vgl. Andreß/Strengmann-Kuhn 1997; Gangl 1998; Wilde 2003; Gebauer et al. 2003; Schneider/Uhlendorff 2005; van Berkel 2007; Gebauer 2007). Die Studien liefern zum Teil widersprüchliche Ergebnisse. In einigen kann ein Einfluss von Sozialtransfers auf das Verhalten am Übergang vom System sozialer Sicherung zum Arbeitsmarkt nachgewiesen werden. So kommen Andreß/Strengmann-Kuhn (1997) zu dem Ergebnis, dass im unteren Einkommensbereich weniger Arbeit angeboten wird als im Durchschnitt. Auch Schneider/Uhlendorff (2005) konnten einen negativen Einfluss eines geringen potenziellen Arbeitseinkommens auf die Wahrscheinlichkeit, den Sozialleistungsbezug zu verlassen, feststellen.

Andere Studien kommen $\mathrm{zu}$ dem Schluss, dass „an der Schnittstelle zwischen Arbeitslosenhilfe und Arbeitsmarkt nicht von einer Armutsfalle gesprochen werden kann“ (Gebauer 2007). Im europaweiten Vergleich besteht weder zwischen Leistungshöhe und Arbeitslosenquote noch zwischen Leistungshöhe und Umfang der Langzeitarbeitslosigkeit ein signifikanter Zusammenhang (vgl. Piachaud 1997, S. 51ff.). Es konnte gezeigt werden, dass der Übergang aus Arbeitslosigkeit in Beschäftigung im Wesentlichen durch individuelle Ressourcen bestimmt wird. Die relative Höhe der Sozialleistungen hat hingegen keinen Einfluss auf den Ausstieg aus dem Sozialtransferbezug (Gangl 1998; Schwarze/Radeschall 2002; Wilde 2003). Nichtpekuniäre Faktoren, beispielsweise das Beratungsverhalten des Sozialamtsmitarbeiters, haben einen höheren Einfluss auf die Ausstiegswahrscheinlichkeit als pekuniäre (Wilde 2003). Studien der dynamischen Armutsforschung kommen zu dem Schluss, dass Menschen auch in prekären Lebenslagen handlungsfähig bleiben und zu einem großen Teil wieder aus Arbeitslosigkeit herausfinden (vgl. Buhr 1995; Gebauer et al 2003; Gebauer 2007). Diese Untersuchungen beziehen sich allerdings alle auf die Zeit vor Hartz IV.

Vor dem Hintergrund der durch die Hartz-IV-Reform geänderten Rahmenbedingungen zeichnet sich weiterhin eine Strukturierung der Bezugsdynamik in kurze und lange, kontinuierliche und 
diskontinuierliche Leistungsperioden ab (Graf/Rudolph 2009). Zwar beschäftigt sich vor allem das Institut für Arbeitsmarkt- und Berufsforschung (IAB) seit der Zusammenlegung von Sozial- und Arbeitslosenhilfe 2005 mit der Dynamik des Sozialtransferbezugs (vgl. u.a. Koch et al. 2009; Graf/Rudolph 2009; Hirseland/ al. 2010), ein Vergleich der Dynamik im Transferbezug vor und nach der institutionellen Intervention wurde aber bisher nicht durchgeführt. ${ }^{2}$

Wir knüpfen an die bisherigen empirischen Prüfungen des Armutsfallentheorems an und entwickeln ein einfaches vergleichendes Design. Untersuchungsgegenstand sind die Verweildauern in Arbeitslosigkeit unter Bezug von unbefristeten sozialpolitischen Lohnersatzleistungen für Arbeitsfähige. Verglichen werden ein Zeitraum A vor und ein Zeitraum B nach Einführung von Hartz IV.

\section{Auswahl der Vergleichsgruppen}

Das Armutsfallentheorem und die Sichtweise des öffentlichen Diskurses stimmen in der Annahme überein, dass Armut ein Langzeitphänomen ist. Der Glaube, dass wer einmal Sozialhilfe bezieht, immer von Sozialhilfe leben wird (vgl. Zwick 1994), wurde dadurch begünstigt, dass die Armutsforschung lange Zeit auf Ramos Lobato 2010 sowie auch Buhr et

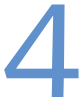

Querschnittdaten angewiesen war. Da auf dieser Grundlage zwischen Personen und Problemlagen nicht unterschieden werden kann, lässt sich jedoch über die tatsächlichen Bezugsdauern von sozialpolitischen Transfers nichts sagen (zur Kritik vgl. Rohwer 1992; Zwick 1994; Buhr 1995; Leisering/Leibfried 1999; Leisering 2008). Abhilfe schaffen Analysen der Bezugsdauern im Längsschnittdesign, wie wir sie hier auf Grundlage des Sozio-oekonomischen Panels (SOEP) vornehmen.

Wir greifen auf Ereignisdaten zum Erwerbsstatus zurück. Diese Daten zu den individuellen Arbeitslosigkeitsepisoden werden mit Angaben zum Sozialleistungsbezug verknüpft, um eine Analyse der individuellen Arbeitslosigkeitsdauern von Transferleistungsbeziehern zu ermöglichen. Im Blickpunkt steht somit eine Untersuchung der Arbeitslosigkeit von Personen, die Sozial- oder Arbeitslosenhilfe (Zeitraum A) und von Personen, die Arbeitslosengeld II (Alg II) (Zeitraum B) erhalten haben. $\mathrm{Zu}$ fragen ist zum einen, wie lange die Arbeitslosigkeitsepisoden von Sozialtransferbeziehern andauern, und zum anderen, ob sich die Episodendauern vor und nach den Hartz-Reformen voneinander unterscheiden. Um den Vergleich durchzuführen, werden die Episodendaten zur Arbeitslosigkeit in zwei Teile zerlegt. Mit Zeitraum A - vor Hartz IV: 1/2002 - 12/2004 - wird die Arbeitslosigkeit bei Sozial- und Arbeitslosenhilfebeziehern untersucht. Zeitraum B - seit Hartz IV: 1/2005 - 12/2007 - bezieht sich auf die Arbeitslosigkeit bei Alg-II-Beziehern.

\section{Tabelle 1: Zusammensetzung der Vergleichsgruppen arbeitsloser} Sozialtransferbezieher vor und nach Hartz IV

\begin{tabular}{|c|c|c|c|}
\hline & $\begin{array}{c}\text { Arbeitslose Sozial-/ } \\
\text { Arbeitslosenhilfebezieher } \\
2002-2004\end{array}$ & $\begin{array}{c}\text { Arbeitslose Arbeitslosengeld- } \\
\text { II-Bezieher } \\
2005-2007\end{array}$ & $\begin{array}{l}{ }^{*} \mathrm{p}(\mathrm{chi} 2) \\
<=0.05\end{array}$ \\
\hline Geschlecht (w) & $47 \%$ & $50 \%$ & * \\
\hline Alter in Jahren (Mittelwert) & 39 & 41 & \\
\hline Migrationshintergrund ${ }^{++}$ & $17 \%$ & $18 \%$ & \\
\hline $\begin{array}{l}\text { Schulbildung } \\
\text { Kein Abschluss } \\
\text { Hauptschule } \\
\text { Realschule } \\
\text { Fachoberschule oder Abitur }\end{array}$ & $\begin{array}{r}15 \% \\
39 \% \\
39 \% \\
7 \%\end{array}$ & $\begin{array}{r}16 \% \\
40 \% \\
35 \% \\
8 \% \\
\end{array}$ & * \\
\hline $\begin{array}{l}\text { Haushaltstyp } \\
\text { Single } \\
\text { Paar ohne Kinder } \\
\text { Paar mit Kindern } \\
\text { Alleinerziehend }\end{array}$ & $\begin{array}{l}31 \% \\
14 \% \\
37 \% \\
18 \%\end{array}$ & $\begin{array}{l}35 \% \\
17 \% \\
29 \% \\
16 \%\end{array}$ & \\
\hline $\begin{array}{l}\text { Gesundheit } \\
\text { weniger gut/schlecht }\end{array}$ & $22 \%$ & $24 \%$ & \\
\hline Fallzahl & 1.639 & 1.253 & \\
\hline $\begin{array}{l}{ }^{+} \text {der } 15 \text { - bis } 65 \text {-jährigen Sozialtrans } \\
{ }^{+} \text {Migrationserfahrung der Eltern. } \\
\text { Quelle: Berechnungen der Autoren }\end{array}$ & $\begin{array}{l}\text { ieher; } \\
\text { ten des SOEP, gewichtet. }\end{array}$ & & LUN \\
\hline
\end{tabular}

$\mathrm{Zu}$ bedenken ist bei dem Vergleich, dass sich die soziodemografische Zusammensetzung der beiden Gruppen aufgrund institutioneller Neuregelungen unterscheidet. Die Hartz-IV-Reform hat zu einer Umschichtung innerhalb des untersten Segments der Einkommensverteilung geführt (Becker/Hauser 2006, S. 102). Einerseits gibt es Personen, die vor der Reform keine Sozialleistungen erhielten, nun aber Alg II erhalten. Anderseits hat sich die Gruppe der Alg-II-Bezieher dadurch vermindert, dass manche früheren Bezieher von Arbeitslosenhilfe wegen geringerer Freibeträge bei der Anrechnung von Vermögen beim Bezug von Alg II nicht mehr als bedürftig gelten (vgl. Brenke 2010, S. 4). Rund $17 \%$ der Arbeitslosenhilfehaushalte haben ab 2005 mangels behördlich anerkannter Bedürftigkeit ihre Ansprüche verloren, zumeist wegen anrechenbarer Einkünfte eines Partners (Koch et al. 2009, S. 39). Die Kreise der arbeitslosen Bezieher unbefristeter Sozialtransfers in den Zeiträumen vor und nach Hartz IV sind also nicht deckungsgleich.

Wir konzentrieren uns auf die Untersuchung von Arbeitslosen, einer Teilgruppe der Sozialtransferbezieher. Damit verzichten wir auf eine Untersuchung des Transferbezugs in Lebenslagen, die Erwerbstätigkeit ausschließen, also zum Beispiel Phasen der Erziehung von Kleinkindern, der Pflege eines Angehörigen oder des Schulbesuchs. Auch eine Untersuchung von sogenannten Aufstockern, die mit ihrer Erwerbstätigkeit nicht ihren Lebensunterhalt verdienen können, wird durch eine Fokussierung auf Arbeitslose ausgeschlossen (vgl. hierzu Dietz et al. 2009).

Tabelle 1 stellt dar, wie sich die beiden so eingegrenzten Untersuchungsgruppen zusammensetzen. Tatsächlich sind die Unterschiede zwischen Sample A und Sample B gering. Der Anteil der arbeitslosen Frauen im Alg-II-Bezug ist signifikant höher als im Vorgängerregime. Arbeitslose Alg-IIBezieher sind im Durchschnitt zwei Jahre älter als arbeitslose Sozial- oder Arbeitslosenhilfebezieher. Der Migrantenanteil ist etwa gleich geblieben und die schulische Bildung differiert ebenfalls nur um wenige Prozentpunkte. Im Alg-II-Bezug befinden

2 Der Grund ist wohl die schlechte Datenlage. Das Sozio-oekonomische Panel (SOEP) ist eine der wenigen Datenquellen, die auf Mikroebene Längsschnittinformationen zum Sozialtransferbezug vor und nach 2005 liefern. 
sich mehr Alleinstehende und Paare ohne Kinder und etwas weniger Alleinerziehende als im Sozial- oder Arbeitslosenhilfebezug. Der Anteil der Paare mit Kindern ist im Alg-II-Bezug geringer: Sie fallen häufig wegen geringerer Freibeträge bei der Anrechnung von Vermögen und Einkünfte der Partner aus dem Transferbezug heraus (vgl. Buhr et al. 2010; Brenke 2010). Etwas mehr arbeitslose Alg-II-Bezieher geben an, eine weniger gute oder schlechte Gesundheit zu haben. Zusammenfassend sind auf Basis dieses deskriptiven Vergleichs, der kaum signifikante Unterschiede ergibt, keine gravierenden Unterschiede in den Arbeitsmarktchancen zwischen den Vergleichsgruppen A und B zu erwarten.

\section{5 \\ Arbeitslosigkeit im alten Sozialregime}

Im ersten Schritt betrachten wir die Verweildauern in Arbeitslosigkeit der Untersuchungsgruppe im Zeitraum A vor Hartz IV. Mithilfe des statistischen Verfahrens der Ereignisdatenanalyse zur Untersuchung von Längsschnittdaten integrieren wir den zeitlichen Faktor von Erwerbsverläufen in unsere Analyse. Es wird die Kaplan-Meier-Schätzung verwendet. Von den Ereignisdaten des SOEP zur Arbeitslosigkeit bei Sozial- und Arbeitslosenhilfebeziehern werden 2.212 Episoden erfasst, von denen 1.824 mit einem Ereignis enden, also nicht rechtszensiert sind. In diesen Fällen fand also ein Übergang von Arbeitslosigkeit in einen anderen Status (Erwerbstätigkeit, Aus- oder Weiterbildung, Rente, Mutterschaft, Wehr- oder Zivildienst) statt.

Wie die Ergebnisse der Schätzung zeigen, scheidet ein hoher Anteil der arbeitslosen Sozial- und Arbeitslosenhilfebezieher innerhalb kurzer Zeit wieder aus der Arbeitslosigkeit aus. Der Median der Episodendauer liegt bei zwölf Monaten. Nach einem Jahr sind $49 \%$ aller begonnenen Arbeitslosigkeits-Episoden beendet, und auch danach kommt die Dynamik des Prozesses nicht zum Stillstand, allerdings verringern sich die Abgangszahlen im Zeitverlauf. Im zweiten Jahr verringert sich die Population arbeitsloser Sozial- und Arbeitslosenhilfebezieher zusätzlich um 20 Prozentpunkte, im dritten Jahr um weitere elf Prozentpunkte und nach vier Jahren verbleiben noch $13 \%$ der Ausgangspopulation in Arbeitslosigkeit. Dieser empirische Befund zeigt, dass ein hoher Anteil der Sozial- und Arbeitslosenhilfebezieher wenig Schwierigkeiten hatte, aus Arbeitslosigkeit herauszufinden. Arbeitslose Sozialleistungsbezieher steckten demnach bereits vor 2005 nicht in einer „Arbeitslosigkeitsfalle“, sondern überwanden zu einem Großteil ihre Beschäftigungslosigkeit. Dieses Ergebnis entspricht Befunden aus früheren Untersuchungen (vgl. Buhr 1995; Gangl 1998; Gebauer et al. 2003; Gebauer 2007).

\section{C \\ Arbeitslosigkeit im neuen Sozialregime}

Grundlage für die Hartz-IV-Reform war die Auffassung, dass die Möglichkeit des unbefristeten Bezugs von Sozialhilfe (als Hilfe zum laufenden Lebensunterhalt) und Arbeitslosenhilfe die Wiederaufnahme von Erwerbstätigkeit behindert. Sollte die Reform gemäß den Annahmen des Armutsfallentheorems Einfluss auf das Verhalten der Sozialtransferbezieher genommen haben, müssten sich bei einer Analyse der Arbeitslosigkeit von Alg-IIBeziehern kürzere Arbeitslosigkeitsphasen ergeben als früher bei den Sozial- und Arbeitslosenhilfebeziehern. Auch hier werden Übergänge von Arbeitslosigkeit in einen anderen Status (Erwerbstätigkeit, Aus- oder Weiterbildung, Rente, Mutterschaft, Wehr- oder Zivildienst) untersucht. Berechnet wird die individuelle Dauer der Arbeitslosigkeit der Personen, die zwischen 2005 und Ende 2007 Alg-II-Bezieher waren. Von den Ereignisdaten des SOEP zur Arbeitslosigkeit bei Alg-II-Beziehern werden 1.665 Episoden erfasst, von denen 1.126 mit einem Ereignis enden.

Die Analyse zeigt, dass auch im Zeitraum $\mathrm{B}$ ein hoher Anteil der arbeitslosen Alg-II-Bezieher innerhalb kurzer Zeit einen Übergang aus Arbeitslosigkeit vollzogen hat. Der Median der Episodendauer liegt bei 13 Monaten. Nach etwas über einem Jahr ist für $50 \%$ aller AlgII-Bezieher die Arbeitslosigkeit beendet, und auch danach kommt die Dynamik des Prozesses nicht zum Stillstand, wenngleich sich die Abgangsraten verringern. Bis Ende des zweiten Jahres verringert sich die Population arbeitsloser Alg-II-Bezieher zusätzlich um 20 Prozentpunkte und zum Ende des dritten Jahres um weitere zehn Prozentpunkte, sodass nach drei Jahren noch $21 \%$ und nach vier Jahren noch $16 \%$ der Ausgangspopulation in Arbeitslosigkeit verbleiben. Dieser empirische Befund zeigt, dass nach wie vor ein relativ hoher Anteil der Bezieher von Sozialleistungen wenig Schwierigkeiten hat, wieder Arbeit zu finden.

\section{7 \\ Vergleich der Arbeits- losigkeit im alten und neuen Regime}

Der Vergleich der Verweildauerkurven der beiden Untersuchungsgruppen ergibt keine markanten Unterschiede (Abbildung 1). Bis zum neunten Verlaufsmonat weichen die Kurven um höchstens einen Prozentpunkt voneinander ab. Im weiteren Zeitverlauf verlassen um 1,3 bis 3,3 Prozentpunkte mehr Sozial- oder Arbeitslosenhilfebezieher als Alg-II-Bezieher die Arbeitslosigkeit. Zusammenfassend kann man sagen: Die Hartz-IV-Reform hat keine deutliche Verkürzung der Arbeitslosigkeitsepisoden gebracht. Trotz des Versuchs, mit Maßnahmen der Aktivierung und verschärften Zumutbarkeits- und Sanktionsregelungen den Übergang vom Sozialleistungsbezug in die Erwerbstätigkeit zu forcieren, trat keine wesentliche Veränderung der Verweildauern von Sozialtransferbeziehern in Arbeitslosigkeit ein.

Mit unserem deskriptiven Untersuchungsdesign konnten wir zeigen, dass sich die Arbeitslosigkeitsepisoden von Beziehern unbefristeter Sozialtransfers drei Jahre vor und nach Einführung des SGB II kaum unterscheiden. Die Frage ist nun, woran das liegt. Wirkt die Reform deshalb nicht, weil ihre Grundlogik, das Armutsfallentheorem, sich empirisch nicht bestätigt? Das ist unsere These. Das Theorem bildet das Handeln von arbeitslosen Sozialtransferbeziehern nur ungenügend $\mathrm{ab}$, weil es soziale Faktoren wie Stigmatisierungseffekte, Statusanreize und Rollenerwartungen, aber auch demografische Prozesse und strukturelle wirtschaftliche Determinanten unberücksichtigt lässt (vgl. Piachaud 1997; Gebauer 2007; Promberger 2008). Es könnte aber auch sein, dass die Hartz-IV-Reform die von ihr intendierten Effekte zwar erzielte, dass aber Konjunk- 
Abb. 1: Vergleich der Verweildauer von Sozialtransferbeziehern in Arbeitslosigkeit vor und nach Hartz-IV

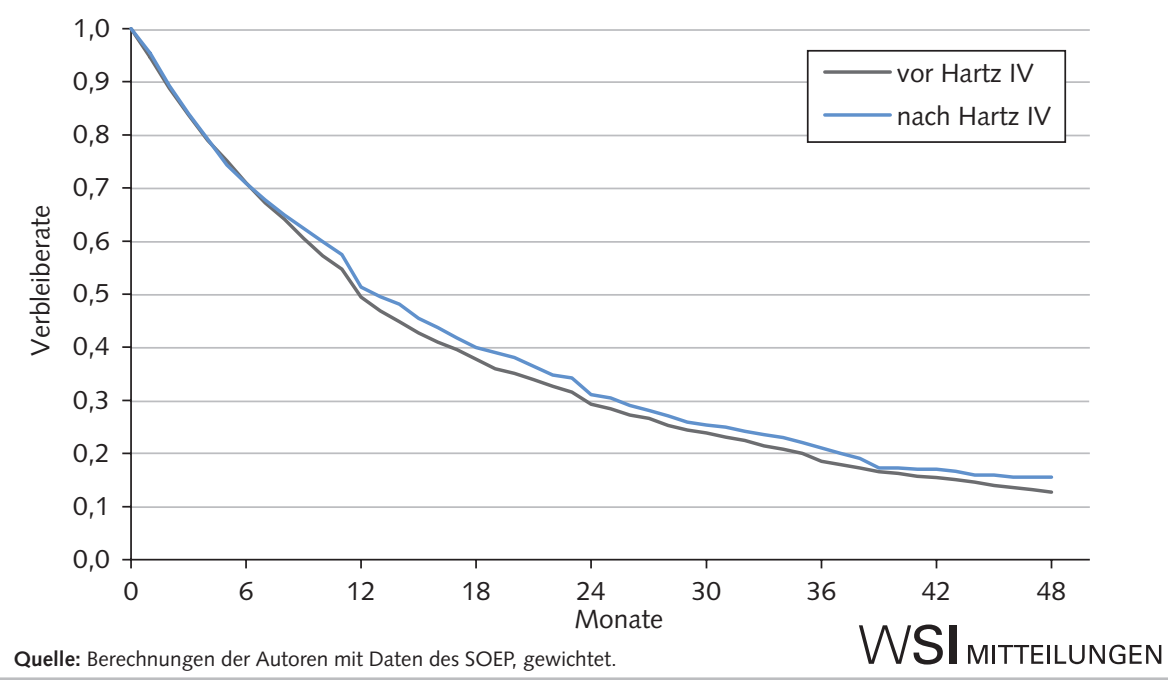

tur und Beschäftigung sowie die teilweise unterschiedliche Zusammensetzung der Vergleichsgruppen ihre positiven Effekte konterkariert haben.

Um die Stabilität unserer Befunde in dieser Hinsicht zu prüfen, leiten wir die Analyse in ein multivariates Verfahren über (Tabelle 2). Von den Faktoren Geschlecht,

Alter, Migrationshintergrund, Bildung und vom Haushaltstyp wird ein Einfluss auf die Verweildauer in Arbeitslosigkeit erwartet (vgl. Buhr 1995; Gebauer et al. 2003; Schneider/Uhlendorff 2005; Gebauer 2007). Ebenso wird angenommen, dass Arbeitslosigkeit einen Hysteresiseffekt hat: Was immer sie ursprünglich verursacht haben mag - sie

\section{Tabelle 2: Einflussfaktoren auf den Abgang aus Arbeitslosigkeit bei Sozialtransferbeziehern - 2002-2007 (Hazardrate, Piecewise- Constant-Exponential-Model)}

\begin{tabular}{|c|c|c|c|c|}
\hline \multirow{2}{*}{$\begin{array}{l}\text { Kovariaten } \\
\text { Geschlecht: weiblich }\end{array}$} & \multicolumn{2}{|c|}{$\begin{array}{c}\text { Modell 1: } \\
\text { Übergänge in Erwerbstätigkeit, } \\
\text { Aus- oder Weiterbildung, } \\
\text { Rente, Mutterschaft, } \\
\text { Wehr- oder Zivildienst }\end{array}$} & \multicolumn{2}{|c|}{$\begin{array}{c}\text { Modell 2: } \\
\text { Übergänge in } \\
\text { Erwerbstätigkeit }\end{array}$} \\
\hline & -.1598 & $* * * *$ & -.3534 & $* * * *$ \\
\hline Alter & -.0242 & $* * * *$ & -.2344 & $* * * *$ \\
\hline Migrationshintergrund & -.0042 & & -.1075 & \\
\hline \multicolumn{5}{|l|}{ Bildung (Referenz: kein Abschluss) } \\
\hline Hauptschulabschluss & .0581 & & .1302 & \\
\hline Realschulabschluss & .2453 & $* * *$ & .3366 & $* * *$ \\
\hline Abitur & .5050 & $* * * *$ & .5633 & $* * * *$ \\
\hline \multicolumn{5}{|l|}{ Haushaltstyp (Referenz: Single) } \\
\hline Paar-Haushalt & .1579 & * & .1506 & \\
\hline Alleinerziehende & -.0258 & & -.0736 & \\
\hline Paare mit Kind(ern) & .1160 & * & .0797 & \\
\hline Gesundheitszustand: weniger gut-schlecht & -.2036 & $* * *$ & -.4203 & $* * * *$ \\
\hline regionale Arbeitslosenquote & -.0051 & & .0041 & \\
\hline $\begin{array}{l}\text { Alg II 2005-2007 (Referenz: } \\
\text { Sozial-/ Arbeitslosenhilfe 2002-2004) }\end{array}$ & -.1382 & $* * *$ & .0547 & \\
\hline 1. Jahr & $-3,3054$ & $* * * *$ & $-3,6863$ & $* * * *$ \\
\hline 2. Jahr & $-3,4881$ & $* * * *$ & $-3,9593$ & $* * * *$ \\
\hline 3. Jahr & $-3,7126$ & $* * * *$ & $-4,2179$ & $* * * *$ \\
\hline 4. Jahr & $-3,8689$ & $* * * *$ & $-4,1851$ & $* * * *$ \\
\hline \multicolumn{5}{|l|}{ Modellgüte } \\
\hline Log likelihood (starting values/final values) & $-4851 /-3927$ & & $-3692 /-3307$ & \\
\hline$x^{2}$ & 0.000 & & 0.000 & \\
\hline
\end{tabular}

wird zu einer Ursache ihrer selbst. Als Indikator für die Arbeitsmarktsituation wird monatsgenau die Arbeitslosigkeit im Bundesland des Sozialtransferbeziehers verwendet. Dabei ist ein negativer Effekt der Arbeitslosenquote auf die individuellen Austrittschancen aus Arbeitslosigkeit zu erwarten. Der Wirkungszeitraum der Hartz-IV-Reform fällt in eine wirtschaftliche Konsolidierungsphase nach den Rezessionen der 1990er Jahre sowie eine Stagnation von 2000 bis 2003 . Seit 2005 hat sich eine konjunkturelle Aufwärtsentwicklung der deutschen Wirtschaft durchgesetzt. Während der Jahresdurchschnittswert der deutschen Arbeitslosenquote im Vergleichszeitraum A zwischen 10,8\% 2002 und 11,7\% 2004 lag, sank die Quote im Vergleichszeitraum B von $13 \%$ im Jahr 2005 auf 10,1 \% im Jahr 2007 (BA 2010). Die veränderte Konjunktur dürfte zu einer Verkürzung der Arbeitslosigkeitsepisoden seit Hartz IV geführt haben. Allerdings haben vom Rückgang der Arbeitslosigkeit in erster Linie Kurzzeitarbeitslose profitiert, während der Aufschwung am Arbeitsmarkt an den Grundsicherungsbeziehern weitgehend vorbei ging (vgl. Buntenbach 2009, S. 254; Brenke 2010).

Die Koeffizienten des Piecewise-Constant-Exponential-Modells beschreiben den Einfluss der gewählten unabhängigen Variablen auf die Hazardrate, sprich die Neigung zu einem Zustandswechsel. In unserem Fall weisen die Koeffizienten also auf einen positiven oder negativen Effekt der Variablen auf die Chancen auf einen Abgang aus Arbeitslosigkeit hin. Es werden zwei Modelle gerechnet. In Modell 1 werden alle Abgänge aus Arbeitslosigkeit als Zustandswechsel betrachtet, also auch Wechsel in Aus- oder Weiterbildung, Rente, Mutterschaft und Wehr- oder Zivildienst. In Modell 2 gelten ausschließlich Abgänge in Erwerbstätigkeit (voll erwerbstätig, Kurzarbeit, Teilzeit, betriebliche Ausbildung, Lehre) als Zustandswechsel. Positive Koeffizienten verweisen auf höhere Chancen auf einen Zustandswechsel gegenüber der jeweiligen Vergleichsgruppe, negative Koeffizienten indizieren geringere Chancen auf einen Zustandswechsel. Ausschlaggebend für die Prüfung unserer Ausgangsthese, dass Hartz IV die Arbeitslosigkeitsepisoden von Sozialtransferbeziehern nicht verkürzt hat (sowie der Ergebnisse des Verweilkurvenvergleichs, die unsere These bestätigen), ist der Effekt des Indikators für den Sozialtransferbezug: Eine 
positive Ausprägung zeigt an, in welchem Umfang der Alg-II-Bezug im Zeitraum 2005-2007 einen positiven Effekt auf die Chancen auf einen Abgang aus Arbeitslosigkeit hat. Eine negative Ausprägung indiziert, dass im Arbeitslosen- oder Sozialhilfebezug 2002-2004 bessere Chancen auf eine Beendigung von Arbeitslosigkeitsphasen bestanden.

In beiden Modellen sinken im Zeitverlauf die Hazardraten. Je länger ein Arbeitsloser unbeschäftigt bleibt, desto geringer sind seine Chancen auf einen $\mathrm{Zu}$ standswechsel. Frauen haben schlechtere Abgangschancen aus Arbeitslosigkeit als Männer. Je älter ein Arbeitsloser ist, desto geringer sind seine Chancen auf einen $\mathrm{Zu}$ standswechsel. Realschulabsolventen und (noch deutlicher) Abiturienten haben signifikant bessere Chancen auf einen Abgang aus Arbeitslosigkeit als Personen ohne Schulabschluss. Arbeitslose, die angeben, eine weniger gute bis schlechte Gesundheit zu haben, haben schlechtere Chancen auf einen Zustandswechsel. Alleinerziehende haben geringere Chancen, die Arbeitslosigkeit hinter sich zu lassen. Allerdings ist dieser Effekt aufgrund geringer Fallzahlen nicht signifikant. Personen, die in Paarhaushalten mit und ohne Kinder leben, haben bessere Abgangschancen aus Arbeitslosigkeit als Personen, die allein leben. Die regionale Arbeitslosenquote hat keinen signifikanten Einfluss auf die Abgangschancen Erwerbsloser aus Arbeitslosigkeit. Besonders in Modell 2 treten humankapitaltheoretisch relevante Produktivitätsfaktoren in den Vordergrund. Personen mit größerer „Marktnähe“ haben bessere Chancen auf einen schnellen Wechsel von Arbeitslosigkeit in Erwerbstätigkeit.
Modell 1 (alle Abgänge aus Arbeitslosigkeit) zeigt: Alg-II-Bezieher haben im neuen Sozialleistungsregime etwas schlechtere Chancen auf einen Austritt aus der Arbeitslosigkeit als Sozial- und Arbeitslosenhilfebezieher im alten Regime. In Modell 2 (Übergänge von Arbeitslosigkeit in Erwerbstätigkeit) ergibt sich kein signifikanter Unterschied zwischen AlgII-Beziehern und Sozial- oder Arbeitslosenhilfebeziehern. Dieses Resultat spricht nicht gegen die Ergebnisse der vergleichenden Verweildaueranalyse.

Wir halten somit fest: Seit der HartzReform haben sich die Arbeitslosigkeitsepisoden der Sozialtransferbezieher nicht verkürzt. Im Gegenteil verweilen Alg-IIBezieher bei Berücksichtigung soziodemografischer Effekte und der Arbeitsmarktsituation eher länger in Arbeitslosigkeit als Sozial- und Arbeitslosenhilfebezieher vor der Einführung des SGB II. Letzteres gilt allerdings nur dann, wenn auch Übergänge aus Arbeitslosigkeit in Nichterwerbstätigkeit in die Analyse einbezogen werden. An der Schnittstelle zwischen Arbeitslosigkeit im Sozialtransferbezug und Arbeitsmarkt hat sich indes nichts geändert.

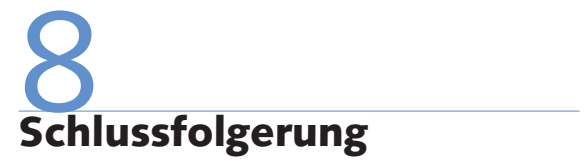

Wir haben uns in dieser Untersuchung der Frage gewidmet, ob es mit der HartzIV-Reform gelungen ist, die allgemein als Problem angesehene Arbeitslosigkeitsfalle zwischen Arbeitsmarkt und dem System sozialer Sicherung zu beseitigen und ausreichende Arbeitsanreize für die Bezieher von Lohnersatzleistungen zu schaffen. Die Antwort hierauf fällt ambivalent aus. Zum einen konnten wir zeigen, dass die Verweildauern von Beziehern sozialpolitischer Transferleistungen in Arbeitslosigkeit nach 2005 tatsächlich überwiegend relativ kurz sind. Dies ist ein Ergebnis, das den Reformintentionen entspricht. Zugleich aber haben wir gezeigt, dass die Verweildauern vor 2005 ähnlich kurz waren. Dieses Ergebnis relativiert die Erfolgsmeldung stark: Als Konsequenz lässt sich festhalten, dass das Problem, um das es der Hartz-Reform zentral ging, nicht existierte; oder, dass es nicht gelungen ist, die Arbeitslosigkeitsdauern weiter zu reduzieren.

Man könnte nun argumentieren, dass die Reform zumindest insoweit erfolgreich war, als sie keinen Schaden verursacht hat. Aber es ist zumindest fraglich, ob dies zutrifft. Es wäre ja denkbar, dass die Umstellung von Sozial- und Arbeitslosenhilfe auf Alg II neben ihrem „Erfolg“ bei der Gestaltung von Arbeitsanreizen und Verweildauern auch soziale und politische Probleme nach sich gezogen hat: eine Zunahme von sozialer Ungleichheit ${ }^{3}$ und Armut, höhere Beschäftigungsunsicherheit sowie Armut trotz Arbeit (working poor), die als soziale Reformkosten zu Buche schlagen und Gerechtigkeitsnormen verletzen. Wir haben eingangs betont, dass dies hier nicht unser Thema ist. Aber wenn die Hartz-IV-Reform gegen gesellschaftlich breit geteilte Gerechtigkeitsvorstellungen verstoßen hat, dann steht diesen Kosten kein Nutzen gegenüber.

3 Die Armutsrisikoquote ist nach der Hartz-Reform von 13 auf $16 \%$ gestiegen und der Gini-Koeffizient als Maßzahl für die Ungleichheit der Einkommensverteilung in einer Gesellschaft wuchs von 2,6 auf 3,0 an (Statistisches Bundesamt 2011). 
Andreß, H.-J./Strengmann-Kuhn, W. (1997): Warum arbeiten, wenn der Staat zahlt?, in: Zeitschrift für Sozialreform 43 (7), S. 505-525 Bane, M.J./Ellwood, D.T. (1986): Slipping into and out of Poverty: The Dynamics of Spells, in: The Journal of Human Resources 21 (1), S. 1-23 Becker, I./Hauser, R. (2006): Verteilungseffekte der Hartz-IV-Reform. Ergebnisse von Simulationsanalysen, Berlin

Berkel, R. van (2007): Social Assistance Dynamics in the Netherlands. Exploring the Sustainability of Independence from Social Assistance via Labour Market Inclusion, in: Social Policy and Society 6 (2), S. 127-139 Brenke, K. (2010): Fünf Jahre Hartz IV. Das Problem ist nicht die Arbeitsmoral, in: Wochenbericht des DIW (6), S. 2-13

Buhr, P. (1995): Dynamik von Armut. Dauer und biographische Bedeutung von Sozialhilfebezug, Opladen

Buhr, P./Lietzmann, T./Voges, W. (2010): Lange Wege aus Hartz IV? Zur Dynamik von Mindestsicherung unter dem Bundessozialhilfegesetz und dem SGB II., in: ZeS Report 15 (1), S. 1-6

Bundesagentur für Arbeit (BA) (2010): Arbeitsmarkt 2009, Amtliche Nachrichten der Bundesagentur für Arbeit 58 (2), Nürnberg Bundesministerium für Wirtschaft und Arbeit (BMWA) (2005): Vorrang für die Anständigen. Gegen Missbrauch, "Abzocke" und Selbstbedienung im Sozialstaat, Berlin

Bundesregierung (2003): Regierungserklärung von Bundeskanzler Schröder am 14. März vor dem Deutschen Bundestag, http://archiv. bundesregierung.de/bpaexport/regierungserklaerung/79/472179/ multi.htm

Bundesregierung (2004): Agenda 2010. Deutschland bewegt sich, Berlin

Buntenbach, A. (2009): Hartz-Reformen. Viel Bewegung - neue Probleme - wenig Fortschritt, in: Seifert, H./Stuck, O. (Hrsg.): Arbeitsmarkt und Sozialpolitik. Kontroversen um Effizienz und soziale Sicherheit,

Wiesbaden, S. 249-264

Dietz, M./Müller, G./Trappmann, M. (2009): Bedarfsgemeinschaften im SGB II. Warum Aufstocker trotz Arbeit bedürftig bleiben, IAB-Kurzbericht (2), Nürnberg

Gangl, M. (1998): Sozialhilfebezug und Arbeitsmarktverhalten. Eine Längsschnittanalyse der Übergänge aus der Sozialhilfe in den Arbeitsmarkt, in: Zeitschrift für Soziologie 27 (3), S. 212-232

Gebauer, R. (2007): Arbeit gegen Armut. Grundlagen, historische Genese und empirische Überprüfung des Armutsfallentheorems, Wiesbaden

Gebauer, R./Petschauer, H./Vobruba, G. (2003): Wer sitzt in der Armutsfalle? Selbstbehauptung zwischen Sozialhilfe und Arbeitsmarkt, Berlin

Gebauer, R./Vobruba, G. (2003): The Open Unemployment Trap. Life at the Intersection of Labour Market and Welfare State. The Case of Germany, in: Journal of Social Policy 32 (4), S. 571-587

Graf, T./Rudolph, H. (2009): Dynamik im SGB II 2006-2007. Viele Bedarfsgemeinschaften bleiben lange bedürftig, IAB-Kurzbericht (5), Nürnberg

Gustafson, B./Müller, R./Negri, N./Voges, W. (2002): Paths through (and out of) Social Assistance, in: Saraceno, C. (Hrsg.): Social Assistance Dynamics in Europe. National and Local Poverty Regimes, Bristol, S. $173-234$

Heikkikilä, M./Keskitalo, E. (2001): Social Assistance in Europe. A Comparative Study on Minimum Income in Seven European countries, Saarijärvi
Hirseland, A./Ramos Lobato, P. (2010): Armutsdynamik und Arbeitsmarkt. Entstehung, Verfestigung und Überwindung von Hilfebedürftigkeit bei Erwerbsfähigen, IAB-Forschungsbericht (3), Nürnberg Knuth, M. (2006): Hartz IV. Die unbegriffene Reform, in: Sozialer Fortschritt 55 (7), S. 160-168

Koch, S./Kupka, P./Steinke, J. (2009): Aktivierung, Erwerbstätigkeit und Teilhabe - Vier Jahre Grundsicherung für Arbeitsuchende, IABBibliothek (315), Bielefeld

Leisering, L. (2008): Dynamik von Armut, in: Huster, E.-U./Boeckh, J./ Mogge-Grotjahn, H. (Hrsg.): Handbuch Armut und soziale Ausgrenzung, Wiesbaden, S. 118-132

Leisering, L./Leibfried, S. (1999): Time of Poverty in Western Welfare States. United Germany in Perspective, Cambridge

Lightman, E./Mitchell, A./Herd, D. (2010): Cycling Off and On Welfare in Canada, in: Journal of Social Policy 39 (4), S. 523-542

Mankiw, N. G. (1999): Grundzüge der Volkswirtschaftslehre, Stuttgart Mises, L. von (1931): Die Ursachen der Wirtschaftskrise, Tübingen Piachaud, D. (1997): Soziale Sicherheit und Abhängigkeit, in: Internationale Revue für Soziale Sicherheit 50 (1), S. 47-63

Promberger, M. (2008): Arbeit, Arbeitslosigkeit und soziale Integration, in: Aus Politik und Zeitgeschichte (40-41), S. 7-15

Rohwer, G. (1992): Einkommensmobilität und soziale Mindestsicherung. Einige Überlegungen zum Armutsrisiko, in: Leibfried, S./Voges, W. (Hrsg.): Armut im modernen Wohlfahrtsstaat, Sonderheft 32 der Kölner Zeitschrift für Soziologie und Sozialpsychologie (KZfSS), Opladen, S. 367-379

Sachverständigenrat zur Begutachtung der gesamtwirtschaftlichen Entwicklung (SVR) (1998): Jahresgutachten 1998/1999, Berlin Saraceno, C. (2002): Social Assistance Dynamics in Europe. National and Local Poverty Regimes, Bristol

Schäfer, H. (2003): Fehlanreize und Reformoptionen in der Arbeitslosenversicherung, in: Sozialer Fortschritt 52 (9), S. 231-233

Schneider, H./Uhlendorff, A. (2005): Transitions from Welfare to Employment: Does the Ratio between Labor Income and Social Assistance Matter?, in: Schmollers Jahrbuch 125, S. 51-61

Schwarze, J./Raderschall, S. (2002): Welfarisation in Deutschland: Werden die Familien abhängig von der Sozialhilfe?, ifb Materialien (8), Bamberg

Sinn, H.-W./Holzner, C./Meister, W./Ochel, W./Werding, M. (2002): Aktivierende Sozialhilfe. Ein Weg zu mehr Beschäftigung und Wachstum, ifo Schnelldienst 55 (9), Sonderausgabe, München Statistisches Bundesamt (2011): Gemeinschaftsstatistik über Einkommen und Lebensbedingungen (EU-SILC), http://www.destatis.de/ jetspeed/portal/cms/Sites/destatis/Internet/DE/Navigation/Statistiken/ WirtschaftsrechnungenZeitbudgets/LebenInEuropa/Tabellen.psml Vobruba, G. (2000): Alternativen zur Vollbeschäftigung,

Frankfurt a. M.

Wilde, J. (2003): Was reizt Sozialhilfeempfänger zum Ausstieg? Eine empirische Untersuchung mit dem Niedrigeinkommenspanel, in: Jahrbücher für Nationalökonomie und Statistik 223, S. 719-742

Zwick, M. (Hrsg.) (1994): Einmal arm - immer arm?, Frankfurt a. M. 